\title{
INCREASE OF ENGINE AIR FILTER ELEMENTS SERVICE INTERVAL FOR MEDIUM AND HEAVY DUTY VEHICLES BY MEANS OF AIR INDUCTION SYSTEM DESIGN OPTIMIZATION.
}

\author{
Alexandre Luchesi de Almeida, Edson Valdomiro de Azevedo, Fernando Jun Yoshino, \\ Marcos Jose Dantas de Oliveira, Wagner Roberta da Silva Trindade \\ MAHLE Metal Leve SA \\ E-mails: alexandre.luchesi.almeida@br.mahle.com, edson.azevedo@br.mahle.com, \\ fernando.yoshino@br.mahle.com,marcos.dantas@br.mahle.com, \\ wagner.trindade@br.mahle.com
}

\begin{abstract}
The engine air filter service interval is one of the bottle necks for heavy and medium duty vehicles maintenance interval. Thus, by increasing its replacement interval, the total ownership costs can be reduced. As desirable side effect, the waste disposal related to the used air filter element change is also positively affected. Based on a systemic approach and numerical simulation tools, the complete vehicle air induction system was redesigned. Computer Fluid Dynamics (CFD) simulations supported the redesign of air/dust pre-separator devices at the air inlet duct and filter element housing. Filter element design, assembling methods and paper bellows parameters were also optimized to further increase filter element dust holding capacity. Overall system performance was evaluated in laboratory tests. In order to confirm the results obtained on laboratory would be valid for field conditions, test standard procedures were compared to data collected in real life severe application. This paper presents numerical simulations and physical tests results of the redesigned system in comparison to traditional air induction systems, bringing breaking through concepts and considering validation as close as real life use conditions.
\end{abstract}

\section{INTRODUCTION}

Engine air cleaners are present in all internal combustion engine automotive vehicles and are responsible for capturing contaminant from the air suctioned by the engine avoiding premature wear to core engine parts. For medium and heavy duty trucks, mostly for applications at high dust concentration countries, the engine air cleaners are generally comprised of a cylindrical filter element placed inside a cylindrical air cleaner housing. The more particles captured by the filter element, the more it will increase the complete system's pressure loss. When the pressure loss reaches a certain pre-defined limit, the filter element must be replaced by a new one.

Especially for commercial vehicles, every maintenance stop corresponds directly to reduced working hours, thus increasing the total ownership costs of the vehicle. Therefore, any solution to increase air filter elements lifetime is highly appreciated by the truck manufacturers. Most direct and logical approach to obtain longer lifetime is to increase filter 
element effective filtration area, especially if this can be achieved without increasing the overall system dimensions. By increasing the effective filtration area, the filter element dust holding capacity (DHC) is also directly improved.

Another approach is directly related to the geometry of the air cleaner housing. Although the cylindrical housing does not offer best packaging solution, if constructed correctly, the airflow inside the housing will be forced to follow a helical path. During this helical movement, the dust particles will be naturally thrown against the inner walls of the housing by centrifugal forces, loosing inertia and therefore being separated from the main airflow. The separated dust is then lately ejected by a correctly placed dust ejection valve. This effect is known as cyclonic effect or simply cyclone.

Due to the cyclonic effect operating method, the pre-separation efficiency, defined by the amount of dust separated and ejected at the ejection valve divided by the total amount of dust injected at the system, is directly related to dust particle size and weight. Simply speaking, the larger and heavier the dust particles, the easier it is to separate them from the main airflow [1]. In this way, characterizing real life dust contaminant and comparing them to laboratory contaminants also brings a better understanding of the potential of the cyclonic effect on real life application.

Finally, for further increasing the pre-separation efficiency, it is sometimes advisable to implement an additional part at the system, generally called air deflector. The air deflector is placed directly between the filter element and air cleaner housing at the inlet area and has the function of further optimize the cyclonic effect. However, all and any new obstacle added between the ambient and the combustion chamber increases overall system pressure loss. The conclusion is that the air cleaner housing, filter element and possible air deflectors must be seen as a whole and must be optimized together, in a systemic approach.

Throughout all the development phases, the new air cleaner concept was compared to a standard air cleaner system commercially available, including the performance test results of both systems in order to evaluate the impact of the optimizations.

\section{CONCEPT DEVELOPMENT}

It is important that the results and conclusions found in this study can be related to improvements in real life. Therefore, the complete development was based on a real platform properly selected taking into consideration its relevance on commercial vehicles market share. As starting point, the system was designed to offer optimized performance with a nominal airflow of $21 \mathrm{~m} / \mathrm{min}$. This airflow correlates to medium duty trucks, with engine displacement of 5 to 7.2 liters.

\subsection{Filter element development}

The filter elements for the discussed applications are generally comprised of a filter paper bellow assembled in a cylindrical geometry to two end discs, one on each side. One of the end discs is completely closed, while the other has a central orifice that it is usually directly assembled to the housing air outlet. The end discs are generally made of polyurethane foam, which also acts as sealing feature between the filter element and the housing, preventing unfiltered air to reach the engine components. 
The drawback of this concept is that, for guaranteeing a sealed assembly between filter element and housing and enough anchoring between paper bellow and end discs, the polyurethane parts must present a minimum height, thus reducing the effective height (i.e. the height of the paper uncovered by the polyurethane foam in the final part) available for the paper bellows and therefore the effective filtration area.

Therefore, for increasing the effective height available for the paper bellows without increasing the overall filter element height, the production concept was completely rethought. The polyurethane discs were replaced by polymeric discs with an attached sealing of polymeric fiber non-woven. The polymeric end discs are then anchored to the paper bellows by plasticizing process. This design concept lead to a direct increase of $3.5 \%$ of effective paper bellows height compared to standard concept.

Parameters such as pitch between each pleat of the paper bellow were also addressed. It is well known that, for each required airflow range, a pleat pitch exists where relation between low pressure loss and high DHC is optimal. As a starting point, an empirical calculation method based on extent database results was used to define these parameters. The parameters were then further optimized through dedicated laboratory tests.

The DHC is generally calculated as the amount of dust retained at the filter element after the system pressure loss achieves a pressure loss increase of $3.0 \mathrm{kPa}$ compared to the initial one. This will be the end-of-test condition used during the complete study.

\subsection{Air cleaner housing development}

Based on previous researches [2], it was aimed to keep the airflow inside housing as laminar as possible. By reducing the amount of turbulence, we guarantee a low initial pressure loss and ensure that the particles will follow the expected helical path.

The main advantage of having a low initial pressure loss related to the housing-filter element system is that air can be more easily suctioned by the engine, thus significantly increasing engine power [3]. Looking from another perspective, in some cases, the air inlet ducts by itself already presents considerably high pressure loss caused by complex geometries or path (in order to attend a specific design requirement or packaging, for example). Having a housing that presents low pressure loss helps achieving a lower pressure loss of the complete system in these cases.

The pressure loss related to the air cleaner housing and filter element system is generally required to be lower than $2.5 \mathrm{kPa}$ for commercial vehicles with a nominal airflow of $21 \mathrm{~m}^{3} / \mathrm{min}$. This value will guide our actions and decisions throughout this study.

\subsection{Air deflector development}

It is known that, beside the function of guiding the air through the desired path inside the housing [4], the air deflector also has major impact on airflow speed inside 
housing. Needless to say that the airflow speed is directly related to pre-separation efficiency [1]: if the airflow is too fast, the air will drag all dust particles all along the way until they are finally suctioned to the filter element; if the airflow is too slow, the cyclonic effect will be very small or inexistent and the dust particles will be directly suctioned to the filter element. Several CFD simulations were performed to qualitative evaluate different air deflectors' geometries and their impact on system pressure loss and airflow speed.

The base geometry of all air deflectors evaluated are the same: a $90^{\circ}$ arc plate long enough to cover the complete air cleaner housing inlet. The air deflector \#1 has a smooth surface on top (some ribs are present only for stiffening purposes); the air deflector \#2 has tall fins with specific angle and curvature, aiming to direct the airflow; the air deflector \#2 also has a smooth surface, but presents a radial wall that acts as a stopper for the airflow after one complete turn inside the housing.

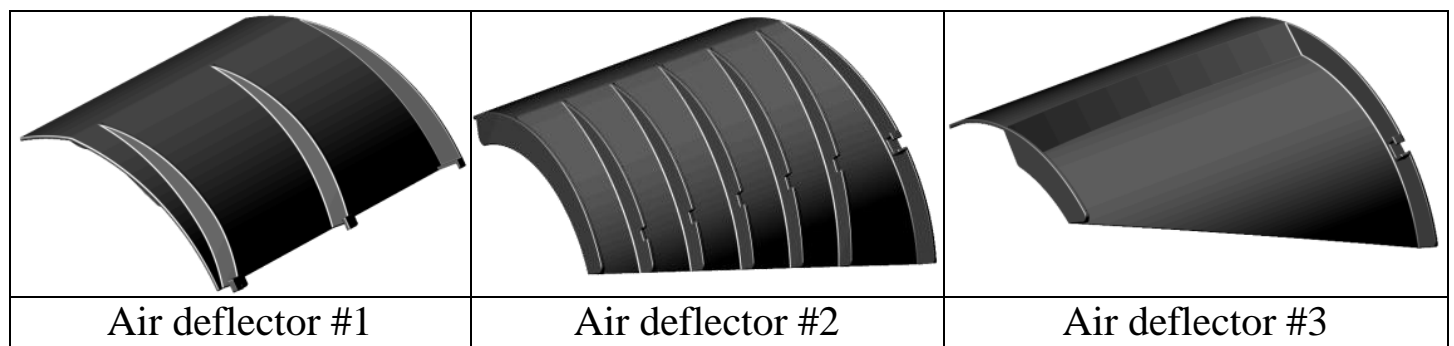

Figure 1: Air deflectors evaluated.

\section{NUMERICAL SIMULATION AND LABORATORY TESTS}

Besides the technical goal of increasing filter element lifetime related to DHC, the technical and economic side of the study was always kept on sight. Therefore, computer fluid dynamics (CFD) simulations and other numeric calculation tools were used to reduce development time and costs. At a second step, the variants studied with most prominent results at simulation were further submitted to laboratory tests to confirm their performance. Although the majority of customers and air cleaner developers submit their systems to tests with ISO Coarse Dust according ISO 12103 standard, for this specific study, some tests will also be performed using ISO Fine Dust, controlled by same international standard.

\subsection{Filter element pleat optimization}

For initially defining the filter element pleat parameters such as pleat height, embossing height and pleat pitch, an internal guideline based on a large number of recorded tests results is used. It is no surprise to confirm that the pleat parameters indicated by the internal guideline are in agreement with what is commonly practiced in market today. As an example, a simple benchmark research indicates that the standard pleat pitch for filter elements of the discussed application varies from 1.8 to $2.3 \mathrm{~mm}$.

Using then an internally developed performance estimation tool, also built based on recorded tests results, the DHC of the proposed filter element, with larger effective filtration area and optimized pleat parameters, is expected to be $11.8 \%$ higher than 
the standard filter element when testing with ISO Coarse Dust and $8.4 \%$ higher when testing with ISO Fine Dust.

DHC Estimation Comparison

\begin{tabular}{c|c|c}
\hline Dust Type & ISO Coarse & ISO Fine \\
\hline $\begin{array}{c}\text { Improvement } \\
\text { (Optimized vs. Standard) }\end{array}$ & $11.8 \%$ & $8.4 \%$ \\
\hline
\end{tabular}

Table 1: DHC estimation comparison.

Aiming to validate the results found on the numerical estimation, the optimized filter element and the standard one were submitted to laboratory tests. The tests were performed according ISO 5011 international standard. Once again, DHC with both ISO Fine and ISO Coarse Dust was recorded.

As presented below, the optimized filter element presented an even higher DHC than estimated, while the standard one presented a lower DHC than estimated. In the end, the optimized filter element presented a DHC up to 33\% higher than the standard version when tested with Coarse dust and $51 \%$ higher when tested with Fine dust.

DHC Laboratory Test Comparison

\begin{tabular}{c|c|c}
\hline Dust Type & ISO Coarse & ISO Fine \\
\hline $\begin{array}{c}\text { Improvement } \\
\text { (Optimized vs. Standard) }\end{array}$ & $33 \%$ & $51 \%$ \\
\hline
\end{tabular}

Table 2: DHC laboratory test comparison.

It is important to say that, as previously discussed, the air cleaner must be seen as a system and, therefore, the optimizations performed at the housing were also relevant for achieving this higher DHC result. Detailed discussions of the results of the system as a whole will be presented later.

\subsection{Air cleaner housing and air deflector optimization}

In order to be able to substitute the solutions already available commercially, the air cleaner housing general dimensions must fall within the expected range for the discussed application. In this way, the outer dimensions of the optimized housing were kept only $2.5 \%$ bigger than the standard housing in the worst case. The housing air inlet and outlet geometry and dimension were kept rigidly the same as the system used as comparison.

On the inside, however, significant changes were tested, including different air deflector geometries. The goal was to achieve at least same pre-separation efficiency of the optimized system without air deflector compared to the standard system and then further offer a solution of increased pre-separation efficiency with addition of an air deflector.

Since the pre-separation concept is based on very complex effects on a micro scale, the prediction of pre-separation efficiency by numerical simulation is made difficult. However, the observation of the flow inside the housing, considering its laminar characteristic and overall velocities, is enough for a well based approach analysis [2]. 
The pictures below show several different air deflector geometries evaluated. The simulations were performed at STAR-CCM+ v11.04.010 solver using finite volume method with steady state flow condition. The turbulent flow was evaluated through a $\mathrm{k}-\varepsilon$ approach. In all cases, the airflow at the air cleaner housing outlet was defined as constant at $21 \mathrm{~m}^{3} / \mathrm{min}$ and the inlet was defined as opened to the environment, with air at $20^{\circ} \mathrm{C}$.

\section{Airflow Path Lines Analysis for Different Air Deflectors' Configuration}

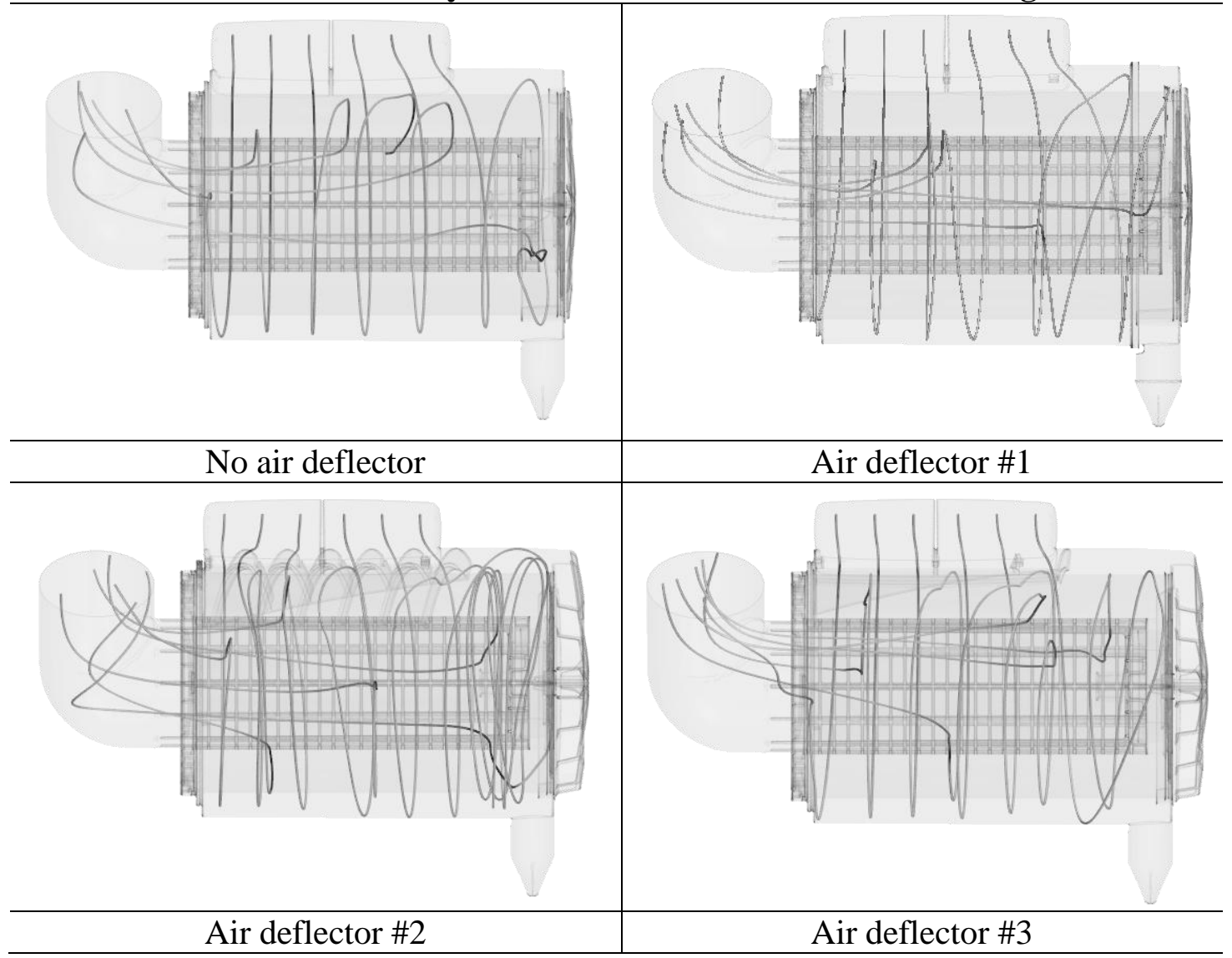

Figure 2: Airflow path lines analysis for different air deflectors' configuration.

Analyzing the pictures above, the statement is that condition with no air deflector and with air deflector \#1 presents most laminar flow, without path lines overlapping each other and most constant airflow speed along the longitudinal section. For instance, the condition with air deflector \#2 and \#3 presented path lines accumulated at the end opposing the clean air duct and clear points of very high or low velocities compared to the average. Therefore, condition without air deflector and with air deflector \#1 should present best pre-separation efficiencies.

Again, in order to validate these estimations in real life, the same geometries were submitted to a laboratory test according ISO 5011, where pressure loss and preseparation with ISO Coarse Dust was evaluated. In this case, the standard air cleaner system was once again used as base for comparison. The table below will show that the optimized air cleaner without air deflector presented virtually exactly same preseparation efficiency compared to the standard air cleaner, with the advantage of presenting an initial pressure loss $31 \%$ lower than the benchmark. The second 
possible configuration, with air deflector \#1, presented virtually same initial pressure loss with an increase of 4 percentage points when compared to the standard. All the pressure drop measured were below the initially specified $2.5 \mathrm{kPa}$.

\begin{tabular}{c|c|c} 
Pressure Loss and Pre-Separation Efficiency Laboratory Test Comparison \\
\hline Test & Pressure Loss & $\begin{array}{c}\text { Pre-Separation } \\
\text { (ISO Coarse) }\end{array}$ \\
\hline $\begin{array}{c}\text { Improvement } \\
\text { (Optimized without air deflector vs. } \\
\text { Standard) }\end{array}$ & $31 \%$ & - \\
\hline $\begin{array}{c}\text { Improvement } \\
\text { (Optimized air deflector \#1 vs. } \\
\text { Standard) }\end{array}$ & - & 4 percentage points \\
\hline
\end{tabular}

Table 3: Pressure loss and pre-separation efficiency laboratory test comparison.

2.3. Synergy between air cleaner housing, air deflector and filter element

As stated since the beginning, in pursuance of having the best overall results, the system must be optimized as a whole. For example, by increasing the effective filtration area of the filter element, the facial velocity at the filter paper (i.e. the airflow rate in cubic meters per second divided by the effective filtration area) is reduced, which in turn further increase the amount of dust the filter paper itself is able to carry by unit of area and reduces the suctioning rate of the air, thus improving the pre-separation effect. In other words, changes in one component of the system might affect performance of other components. If this synergy is understood correctly, the overall results presented by the system are maximized.

Going into this direction, the table below presents the results of total dust retained and/or eliminated by the system (in this case, called simply "Total System DHC" or "total DHC") as evaluated in a test according ISO 5011 using ISO Coarse Dust. The optimized system without air deflector presented a total DHC 33\% higher than the standard system. When the air deflector \#1 was added, the optimized system presented $48 \%$ higher total DHC than the standard part.

Total DHC Laboratory Test Comparison

\begin{tabular}{c|c}
\hline Dust & ISO Coarse \\
\hline $\begin{array}{c}\text { Improvement } \\
\text { (Optimized without air deflector vs. Standard) }\end{array}$ & $33 \%$ \\
\hline $\begin{array}{c}\text { Improvement } \\
\text { (Optimized with air deflector \#1 vs. Standard) }\end{array}$ & $48 \%$ \\
\hline
\end{tabular}

Table 4: Total DHC laboratory test comparison.

\section{TEST DUST COMPARED TO REAL CONDITION DUST}

Throughout the complete study, both optimized and standard air cleaner system were submitted to tests with controlled ISO dusts to evaluated filtration performance. The use of ISO dusts on such tests is important to evaluate specific features at the system and, mostly, to be able to compare test results done in a different occasion and laboratory. However, the 
contaminants found on real condition are much more heterogeneous and the correlation between real dust and lab test dust is not easily addressed [5].

As discussed earlier, the size of the particles is a key factor on both pre-separation efficiency, and filter element DHC. Therefore, characterizing the real condition dust may lead to a system not designed to perform well on laboratory, but also in real life, offering real benefits to customers and users.

It makes sense to start the evaluation at a field where the need for higher lifetime is critical [2] and even small improvements in pre-separation and filter element DHC corresponds to a considerable reduction in total ownership costs. That said, the study focused on evaluating dust on severe, off-road conditions, more specifically a harvester operating in a sugar cane field.

Since the air intake ducts usually are located close to the top of the vehicle cabin, aiming to get as much as fresh air as possible, collecting dust directly from the ground may not be representative. With that in mind, it was made sure that the sample collected dust that was already suspended in the air by the movements of the vehicle and natural wind in a height close to the air inlet duct.

The chart below compares the particle size distribution of the dust collected on the conditions explained above with the ISO Fine and ISO coarse dust.

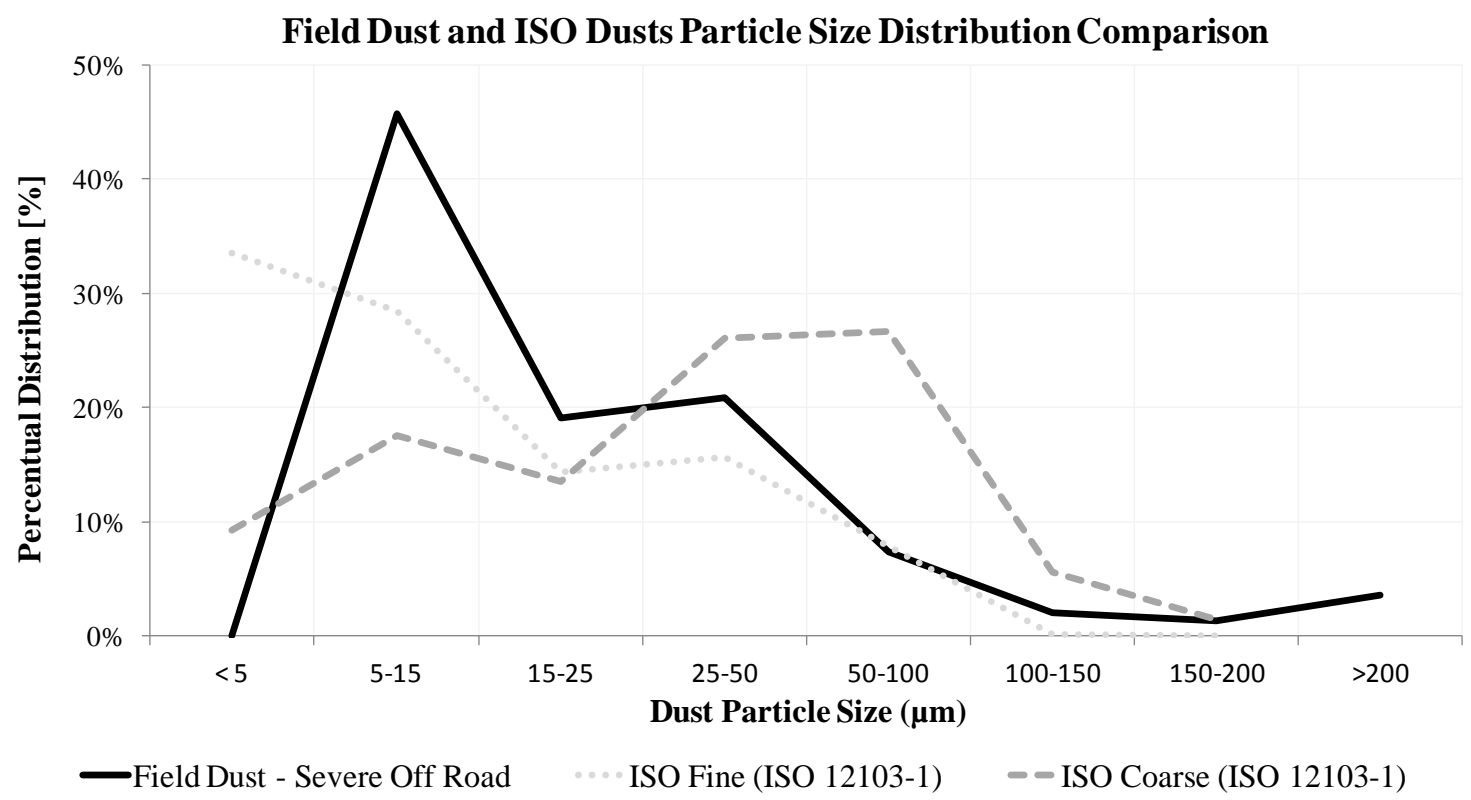

Figure 3: Field dust and ISO dusts particle size distribution comparison

The results show that the dust that is mostly susceptible to be suctioned by the air inlet duct and, therefore, reach the air cleaner housing and filter element, correlates better with ISO Fine Dust than ISO Coarse Dust, with more particles with size smaller than $25 \mu \mathrm{m}$. With that said, contrary to what is generally practiced by commercial vehicles and air cleaner systems manufacturers, for having a better correlation between laboratory and field tests, the efforts on dust retention improvement by air cleaner systems for severe off-road conditions should focus on testing the system with ISO Fine Dust. 
Although unfortunately the total DHC of the optimized system with ISO Fine Dust was not recorded on this study, the results of filter element DHC with same dust and the overall preseparation efficiency indicates that the optimized system should present a total DHC of at least $51 \%$ higher than the standard system without air deflectors, with this result tending to increase even further when tested with the air deflector \#1.

\section{CONCLUSIONS}

A novel air cleaner system for medium duty commercial vehicles was developed focused on optimization of filter element, air cleaner housing, air deflector in a systemic approach. The initial results obtained on numerical simulation and other estimation tools were replicated with good correlation on laboratory tests. The synergy between the parts was made clear when the optimized system without air deflector presented total DHC 33\% higher than the standard system and the optimized system with air deflector \#1 presented total DHC $48 \%$ higher. From the real condition dust characterization, it was concluded that for a better comparison between real life performance on severe off-road applications and laboratory tests, ISO Fine Dust should be used. As next steps, the total DHC of the system with ISO Fine Dust should be evaluated in laboratory, and then the same system should be submitted to a real-life test to further comprehend the correlation between both tests conditions.

\section{REFERENCES}

[1] PEUKERT, W.; WADENPOHL, C. Industrial separation of fine particles with difficult dust properties. Powder Technology, volume 118, issues 1-2, pages 136-148, 8 August 2001. [2] AMARAL, T., ZELLER, a. Air Cleaner Performance Improvement through Multicyclone. SAE Technical Paper 2013-36-0389, 07 October 2013.

[3] BISHOP, I. Effect of Design Variables on Friction and Economy. SAE Technical Paper 640807, 01 January 1964.

[4] CHENG, D., SAAD, M. Application of Rotational Transformation of Flow to

Improve Engine Air Induction System. SAE Technical Paper 930060, 01 March 1993.

[5] HAN, S., YOUN, J. Characterization of PM10 and PM2.5 source profiles for resuspended road dust collected using mobile sampling methodology. Atmospheric Environment, volume 45, issue 20, pages 3343-3351, June 2011.

\section{DEFINITIONS/ABREVIATIONS}

\section{CFD}

\section{DHC}

\section{Initial Pressure Loss}

\section{Pre-separation Efficiency}

Computer Fluid Dynamics

\section{Dust Holding Capacity}

Difference in pressure between inlet and outlet with filter element in new condition.

Mass of dust separated by cyclonic effect and ejected divided by total dust injected 